\title{
DUALITY WITHOUT CONSTRAINT QUALIFICATION IN NONSMOOTH OPTIMIZATION
}

\author{
S. NOBAKHTIAN
}

Received 7 May 2005; Revised 26 July 2005; Accepted 1 January 2006

We are concerned with a nonsmooth multiobjective optimization problem with inequality constraints. In order to obtain our main results, we give the definitions of the generalized convex functions based on the generalized directional derivative. Under the above generalized convexity assumptions, sufficient and necessary conditions for optimality are given without the need of a constraint qualification. Then we formulate the dual problem corresponding to the primal problem, and some duality results are obtained without a constraint qualification.

Copyright $\odot 2006$ Hindawi Publishing Corporation. All rights reserved.

\section{Introduction}

A multiobjective problem is a problem where two or more objective functions are to be minimized on an implicitly constrained feasible set. In such a problem for optimality conditions and duality results, we often deal with constraint qualifications. A constraint qualification assumes some regularity of the constraint functions near the optimal solution, in particular to exclude a cusp on the boundary of the feasible region. In some approaches to multiobjective optimization problems, the necessary conditions for efficiency are derived under the same constraint qualifications as in nonlinear programming with a scalar-valued objective function. Constraint qualifications are often assumed but do not always hold. Some of the well-known qualifications are the Kuhn-Tucker qualification, Slater qualifications [1], and Mangasarian-Fromovitz qualification [7].

Weir and Mond [8] defined dual problems for the scalar-valued programming problems where the usual convexity requirement for duality was relaxed and a constraint qualification was not needed. They established their results using differentiability. Then in [9] Mond and Weir extended their results for multiobjective programs. They defined dual problems for a convex multiobjective programming and a convex/concave multiobjective fractional programming problem where the functions involved are not assumed to be differentiable and where a constraint qualification is not required. Egudo et al. [5] have defined dual problems for differentiable multiobjective programming problems where a 
constraint qualification is not assumed; their approach was different from that in [9] by focusing on efficiency rather than proper efficiency. That approach has the advantage of being suitable to define duals to nonconvex programming problems.

In this paper, we will introduce certain generalized convex functions and then necessary and sufficient optimality conditions are obtained for nondifferentiable multiobjective problems without the need of a constraint qualification. We also define dual problems corresponding to primal problems and then we prove some duality results without the need of a constraint qualification and differentiability. In our approach also the usual convexity requirement for the functions is relaxed.

\section{Definitions and preliminaries}

We consider the following multiobjective problem:

$$
\begin{gathered}
\min f(x)=\left(f_{1}(x), \ldots, f_{m}(x)\right) \\
\text { s.t. } \\
g(x)=\left(g_{1}(x), \ldots, g_{p}(x)\right) \leq 0, \quad x \in C,
\end{gathered}
$$

where $C$ is a convex set and $f: \mathbb{R}^{n} \rightarrow \mathbb{R}^{m}$ and $g: \mathbb{R}^{n} \rightarrow \mathbb{R}^{p}$ are locally Lipschitz functions. The index sets are $M=\{1,2, \ldots, m\}, P=\{1,2, \ldots, p\}$. We denote the feasible set $\{x \in C \mid$ $\left.g_{i}(x) \leq 0, i=1, \ldots, p\right\}$ by $F$. Let $I\left(x^{*}\right)=\left\{i \in P \mid g_{i}\left(x^{*}\right)=0\right\}$ denote the index set of active constraints at $x^{*}$. The minimal index set of active constraints for $F$ is denoted by

$$
I^{=}=\left\{i \in P: x \in F \Longrightarrow g_{i}(x)=0\right\}
$$

We also denote

$$
I^{<}\left(x^{*}\right)=I\left(x^{*}\right) \backslash I^{=}=\left\{i \in I\left(x^{*}\right): \exists x_{i} \in F \text { s.t. } g_{i}\left(x_{i}\right)<0\right\}
$$

For a fixed $r \in M$ and $x^{*} \in \mathbb{R}^{n}$, denote

$$
\begin{gathered}
M^{r}=M \backslash\{r\}, \\
F^{r}\left(x^{*}\right)=\left\{x: f_{i}(x) \leq f_{i}\left(x^{*}\right), i \in M^{r}\right\}, \\
M^{r=}\left(x^{*}\right)=\left\{i \in M^{r}: f_{i}(x)=f_{i}\left(x^{*}\right), \forall x \in F^{r}\left(x^{*}\right)\right\} .
\end{gathered}
$$

We denote $C^{*}=\left\{u \in \mathbb{R}^{n}, u^{t} x \geq 0, \forall x \in C\right\}$ for the polar set of an arbitrary set $C \subset \mathbb{R}^{n}$.

For a nonempty subset $C$ of $\mathbb{R}^{n}$, we denote by $\operatorname{co}(C)$, cone $(C)$, and $C^{*}$ the convex hull of $\mathrm{C}$, the cone generated by $C$, and the dual cone of $C$, respectively. Further, $N_{C}\left(x^{*}\right)$ denotes the normal cone to $C$ at $x^{*}$, defined by

$$
N_{C}\left(x^{*}\right)=\left\{d \in \mathbb{R}^{n}:\left\langle d, x-x^{*}\right\rangle \leq 0, \forall x \in C\right\}
$$

clearly, $\left(C-x^{*}\right)^{*}=-N_{C}\left(x^{*}\right)$. 
Definition $2.1[3,4]$. The generalized Clarke directional derivative of a locally Lipschitz function $f$ at $x$ in the direction $d$ is defined by

$$
f^{c}(x ; d):=\limsup _{\substack{y \rightarrow x \\ t \downarrow 0}} \frac{f(y+t d)-f(y)}{t} .
$$

The Clarke generalized subgradient of a locally Lipschitz function $f$ at $x$ is defined by

$$
\partial_{c} f(x):=\left\{\xi \in \mathbb{R}^{n}: f^{c}(x ; d) \geq\langle\xi, d\rangle, \forall d \in \mathbb{R}^{n}\right\}
$$

We now recall the following result from [4].

Lemma 2.2. Let $f$ be a locally Lipschitz function, and $x \in \operatorname{dom} f$. Then for all $d$ in $\mathbb{R}^{n}$,

$$
f^{c}(x ; d)=\max \left\{\langle\xi, d\rangle: \xi \in \partial_{c} f(x)\right\},
$$

and $\partial_{c} f(x)$ is a nonempty, convex, and compact set.

Definition 2.3. A point $\bar{x} \in F$ is said to be an efficient solution of the minimum problem $(M P)$ if there exists no $x \in F$ such that $f_{i}(x)<f(\bar{x})$ for some $i \in M$ and $f_{j}(x) \leq f_{j}(\bar{x})$ for all $j \in M$.

Definition 2.4. Let $f: \mathbb{R}^{n} \rightarrow \mathbb{R}$ be a locally Lipschitz function. Then

(i) it is said to be generalized convex at $x$ if for any $y$,

$$
f(y)-f(x) \geq\langle\xi, y-x\rangle, \quad \forall \xi \in \partial_{c} f(x),
$$

(ii) it is said to be generalized quasiconvex at $x$ if for any $y$, such that $f(y) \leq f(x)$,

$$
\langle\xi, y-x\rangle \leq 0, \quad \forall \xi \in \partial_{c} f(x)
$$

(iii) it is said to be generalized strictly quasiconvex at $x$ if for any $y$, such that $f(y) \leq$ $f(x), y \neq x$,

$$
\langle\xi, y-x\rangle<0, \quad \forall \xi \in \partial_{c} f(x) .
$$

\section{Necessary and sufficient optimality conditions}

Consider the following nonlinear programming problem:

$$
\begin{gathered}
\min f_{0}(x) \\
\text { s.t. } \\
g_{i}(x) \leq 0, \quad i \in P, x \in C,
\end{gathered}
$$

where $f_{0}$ and $g_{i}, i \in P$, are scalar, locally Lipschitz functions and $C$ is a convex set.

To prove the next results we need the following theorems for the nonlinear program $(S P)$. 
Lemma 3.1. If a feasible point $x^{*}$ of $(S P)$ is optimal, then the following intersection is empty:

$$
\text { cone }\left(C-x^{*}\right) \cap F_{0} \cap G_{0}
$$

where $F_{0}=\left\{d: f_{0}^{c}\left(x^{*} ; d\right)<0\right\}$ and $G_{0}=\left\{d: g_{j}^{c}\left(x^{*} ; d\right)<0, j \in I\left(x^{*}\right)\right\}$.

Proof. Suppose that there exists $d \in \operatorname{cone}\left(C-x^{*}\right)$ such that $d \in F_{0} \cap G_{0}$. Then there exist sufficiently small positive numbers $\lambda_{1}, \lambda_{2}$, and $\lambda_{3}$ such that $x^{*}+\alpha d \in C$, whenever $0<$ $\alpha \leq \lambda_{1}$ and

$$
\begin{aligned}
& f_{0}\left(x^{*}+\alpha d\right)<f_{0}\left(x^{*}\right), \quad \forall \alpha \in\left(0, \lambda_{2}\right), \\
& g_{j}\left(x^{*}+\alpha d\right)<g_{j}\left(x^{*}\right), \quad \forall \alpha \in\left(0, \lambda_{3}\right) .
\end{aligned}
$$

Since $g_{j}\left(x^{*}\right)<0$, for $j \notin I\left(x^{*}\right)$, and by continuity of $g_{j}$, there exists $\lambda_{4}>0$ such that

$$
g_{j}\left(x^{*}+\alpha d\right)<0, \quad \forall \alpha \in\left(0, \lambda_{4}\right) .
$$

Now, let $\lambda=\min \left\{\lambda_{1}, \ldots, \lambda_{4}\right\}$. This contradicts the optimal solution of $(S P)$ at $x^{*}$.

Remark 3.2. Lemma 3.1 implies that the following intersection is also empty:

$$
\text { cone }\left(C-x^{*}\right) \cap\left\{d: f_{0}^{c}\left(x^{*} ; d\right)<0\right\} \cap\left\{d: g_{j}^{c}\left(x^{*} ; d\right)<0 j \in I\left(x^{*}\right) \backslash I^{=}\right\}=\varnothing .
$$

Theorem 3.3. If a feasible point $x^{*}$ of (SP) is optimal and $g_{i}, i \in P$, are generalized strictly quasiconvex at $x^{*}$, then there exist a vector $d \in-N_{C}\left(x^{*}\right)$ and nonnegative scalars $\lambda_{i}, i \in$ $I^{<}\left(x^{*}\right)$, such that

$$
d \in \partial_{c} f_{0}\left(x^{*}\right)+\sum_{i \in I^{<}\left(x^{*}\right)} \lambda_{i} \partial_{c} g_{i}\left(x^{*}\right)
$$

Proof. By applying Lemma 3.1, the system

$$
d \in \text { cone }\left(C-x^{*}\right), \quad f_{0}^{c}\left(x^{*} ; d\right)<0, \quad g_{j}^{c}\left(x^{*} ; d\right)<0, \quad \forall j \in I^{<}\left(x^{*}\right),
$$

has no solution. Then by Lemma 2.2 the system

$$
d \in \text { cone }\left(C-x^{*}\right), \quad \max _{\xi \in A}\langle\xi, d\rangle<0
$$

has no solution, where $A=\partial_{c} f_{0}\left(x^{*}\right) \cup\left(\bigcup_{j \in I^{<}\left(x^{*}\right)} \partial_{c} g_{j}\left(x^{*}\right)\right)$. Since $A$ is a nonempty compact set and cone $\left(C-x^{*}\right)$ is convex, by the alternative theorem [6, Theorem 3.1],

$$
o \in-\left(C-x^{*}\right)^{*}+\operatorname{co}(A)
$$

That is to say, there exist $\lambda_{0} \geq 0, \lambda_{j} \geq 0, j \in I^{<}\left(x^{*}\right)$, and

$$
\xi_{j} \in \partial_{c} g_{j}\left(x^{*}\right), \quad j \in I^{<}\left(x^{*}\right), \quad \xi_{0} \in \partial_{c} f_{0}\left(x^{*}\right), \quad d \in-N_{C}\left(x^{*}\right)
$$

such that

$$
\lambda_{0}+\sum_{j \in I^{<}\left(x^{*}\right)} \lambda_{j}=1, \quad d=\lambda_{0} \xi_{0}+\sum_{j \in I^{<}\left(x^{*}\right)} \lambda_{j} \xi_{j} .
$$


Observe that when $I^{=} \neq \varnothing$, we have $\lambda_{0} \neq 0$. Otherwise, if $\lambda_{0}=0$, then $\sum_{i \in I^{<}\left(x^{*}\right)} \lambda_{i}>0$ and $d=\sum_{i \in I^{<}\left(x^{*}\right)} \lambda_{i} \xi_{i}$, which implies that the system

$$
d \in \text { cone }\left(C-x^{*}\right), \quad \max _{\xi \in B}\langle\xi, d\rangle<0
$$

has no solution, where $B=\bigcup_{i \in I^{<}\left(x^{*}\right)} \partial_{c} g_{i}\left(x^{*}\right)$. On the other hand, since $I^{=} \neq \varnothing$, for each $i \in I^{<}\left(x^{*}\right)$, there exists $x_{i} \in F$ such that $g_{i}\left(x_{i}\right)<0=g_{i}\left(x^{*}\right)$. By generalized strictly quasiconvexity of $g_{i}, i \in P$, there exists $d_{i} \in \operatorname{cone}\left(C-x^{*}\right)$, such that

$$
g_{i}^{c}\left(x^{*} ; d_{i}\right)<0
$$

Hence, for $\bar{d}:=\left(1 / \operatorname{card}\left(I^{<}\left(x^{*}\right)\right)\right) \sum_{i \in I^{<}\left(x^{*}\right)} d_{i}$, we have

$$
g_{i}^{c}\left(x^{*} ; \bar{d}\right)<0, \quad \forall i \in I^{<}\left(x^{*}\right) .
$$

This contradicts the alternative theorem [6, Theorem 3.1] and hence $\lambda_{0}=1$ may be assumed and this completes the proof.

Remark 3.4. If $I^{=}=\varnothing$, then the constraints of the problem satisfy a constraint qualification. In this case, the optimality condition is simplified.

Theorem 3.5. Let $x^{*}$ be a feasible point of (SP). Assume that condition (I) holds at $x^{*}$. If the functions $f_{0}, g_{i}, i \in P$, are generalized convex at $x^{*}$, then $x^{*}$ is a minimum of (SP).

Proof. Let $x \in F$ be any feasible point of $(S P)$. Then from $(I)$, for each $d \in \operatorname{cone}\left(C-x^{*}\right)$,

$$
\left\langle\xi_{0}+\sum_{i \in I^{<}\left(x^{*}\right)} \lambda_{i} \xi_{i}, d\right\rangle \geq 0
$$

for some $\xi_{0} \in \partial_{c} f_{0}\left(x^{*}\right), \xi_{i} \in \partial_{c} g_{i}\left(x^{*}\right), i \in I^{<}\left(x^{*}\right)$. Since each function is generalized convex at $\left(x^{*}\right), x$ is feasible for $(S P)$, and $\sum_{i \in I^{<}\left(x^{*}\right)} \lambda_{i} g_{i}\left(x^{*}\right)=0$, then

$$
\begin{aligned}
f_{0}(x)-f_{0}\left(x^{*}\right) \geq\left\langle\xi_{0}, x-x^{*}\right\rangle & \geq\left\langle-\sum_{i \in I^{<}\left(x^{*}\right)} \lambda_{i} \xi_{i}, x-x^{*}\right\rangle \\
& \geq-\sum_{i \in I^{<}\left(x^{*}\right)} \lambda_{i} g_{i}(x)+\sum_{i \in I^{<}\left(x^{*}\right)} \lambda_{i} g_{i}\left(x^{*}\right) \\
& =-\sum_{i \in I^{<}\left(x^{*}\right)} \lambda_{i} g_{i}(x) \geq 0 .
\end{aligned}
$$

Hence $x^{*}$ is an optimal solution for $(S P)$.

Now we consider problem $(M P)$. The following results is a well-known characterization of the efficient solutions of $(M P)$. 
Lemma 3.6 [2]. A feasible solution $x^{*}$ in $(M P)$ is an efficient solution if and only if $\left(x^{*}\right)$ solves the scalar optimization problem

$$
\begin{gathered}
\min f_{r}(x) \\
\text { s.t. } \\
f_{i}(x) \leq f_{i}\left(x^{*}\right), \quad i \in M^{r}, \\
g_{j}(x) \leq 0, \quad j \in P, x \in C,
\end{gathered}
$$

for each $r=1,2, \ldots, m$.

Theorem 3.3 and Lemma 3.6 can be used to derive necessary and sufficient optimality conditions without constraint qualification for the multiobjective programming problem $(M P)$.

THeORem 3.7. If $x^{*}$ is an efficient solution for $(M P)$ and $g_{i}, i \in P$, are generalized strictly quasiconvex at $x^{*}$, then there exist scalars $\lambda_{i}^{*}>0, i \in M$, with $\sum_{i=1}^{m} \lambda_{i}^{*}=1$, and $\mu_{i}^{*} \geq 0, i \in$ $I^{<}\left(x^{*}\right)$, such that

$$
0 \in \sum_{i \in M} \lambda_{i}^{*} \partial_{c} f_{i}\left(x^{*}\right)+\sum_{i \in I^{<}\left(x^{*}\right)} \mu_{i}^{*} \partial_{c} g_{i}\left(x^{*}\right)+N_{C}\left(x^{*}\right)
$$

Conversely, if (3.15) is satisfied and for every $f_{i}, g_{i}$ are generalized convex at $x^{*}$, then $x^{*}$ is an efficient solution for (MP).

Proof. Necessity. Since $x^{*}$ is an efficient solution for $(M P)$, then, by Lemma 3.6, $x^{*}$ is an optimal solution for each $\left(P_{r}\left(x^{*}\right)\right), r \in M$. Therefore, by Theorem 3.3, there exist $\lambda_{r i}^{*} \geq$ $0, i \in M^{r}, \mu_{r j}^{*} \geq 0, j \in I^{<}\left(x^{*}\right)$, such that

$$
0 \in \partial_{c} f_{r}\left(x^{*}\right)+\sum_{i \in M^{r} \backslash M_{\underline{\underline{r}}}\left(x^{*}\right)} \lambda_{r i}^{*} \partial_{c} f_{i}\left(x^{*}\right)+\sum_{j \in I^{<}\left(x^{*}\right)} \mu_{r j}^{*} \partial_{c} g_{j}\left(x^{*}\right)+N_{C}\left(x^{*}\right)
$$

for each $r \in M$. Now summing the above over $r \in M$, scaling appropriately, and using the properties of $N_{C}\left(x^{*}\right)$ imply the necessary condition.

Sufficiency. Suppose that $x^{*}$ is not efficient for $(M P)$ and (3.15) holds. Then there exist

$$
\begin{gathered}
\xi_{i} \in \partial_{c} f_{i}\left(x^{*}\right), \quad i=1,2, \ldots, m, \quad \eta_{i} \in \partial_{c} g_{i}\left(x^{*}\right), \quad i \in I^{<}\left(x^{*}\right), \\
d \in-N_{C}\left(x^{*}\right), \quad \lambda_{i}^{*}>0, \quad i \in M, \quad \mu_{i}^{*} \geq 0, \quad i \in I^{<}\left(x^{*}\right),
\end{gathered}
$$

such that

$$
\sum_{i \in M} \lambda_{i}^{*} \xi_{i}+\sum_{i \in I^{<}\left(x^{*}\right)} \mu_{i}^{*} \eta_{i}=d
$$

and there exits $u \in F$ such that

$$
\begin{gathered}
f_{i}(u)<f_{i}\left(x^{*}\right), \quad \text { for some } i, \\
f_{j}(u) \leq f_{j}\left(x^{*}\right), \quad \forall j \in M .
\end{gathered}
$$


Hence

$$
\sum_{i \in M} \lambda_{i}^{*} f_{i}(u)<\sum_{i \in M} \lambda_{i}^{*} f_{i}\left(x^{*}\right)
$$

Since the $f_{i}, i \in M$ and $g_{i}, i \in P$ are generalized convex, it follows that

$$
\begin{aligned}
\sum_{i \in M} \lambda_{i}^{*} f_{i}(u)-\sum_{i \in M} \lambda_{i}^{*} f_{i}\left(x^{*}\right) & \geq\left(u-\left(x^{*}\right)\right)^{t} \sum_{i \in M} \lambda_{i}^{*} \xi_{i} \\
& =\left(u-x^{*}\right)^{t}\left(d-\sum_{i \in I^{<}\left(x^{*}\right)} \mu_{i}^{*} \eta_{i}\right) \\
& \geq-\left(u-x^{*}\right)^{t} \sum_{i \in I^{<}\left(x^{*}\right)} \mu_{i}^{*} \eta_{i} \\
& \geq-\sum_{i \in I^{<}\left(x^{*}\right)} \eta_{i} g_{i}(u)+\sum_{i \in I^{<}\left(x^{*}\right)} \eta_{i} g_{i}\left(x^{*}\right) \\
& =-\sum_{i \in I^{<}\left(x^{*}\right)} \eta_{i} g_{i}(u) \geq 0,
\end{aligned}
$$

which contradicts (3.20), hence $x^{*}$ is an efficient solution.

\section{Duality}

We now associate the Wolf-type vector dual [10] to the primal problem $(M P)$ :

$$
\begin{gathered}
\max \bar{f}(u)=\left(f_{1}(u)+y^{t} g(u), \ldots, f_{m}(u)+y^{t} g(u)\right) \\
\text { s.t. } \\
0 \in \sum_{i \in M} \lambda_{i} \partial_{c} f_{i}(u)+\sum_{i \in P} y_{i} \partial_{c} g_{i}(u)+N_{C}(u), \\
y_{i} \geq 0, \quad i=1,2, \ldots, p, \\
\lambda_{i}>0, \quad i=1,2, \ldots, m, \\
\sum_{i \in M} \lambda_{i}=1, \quad g_{I}(u)=0 .
\end{gathered}
$$

Here $F_{D}$ denotes the set of feasible solutions to $(D M)$ and $g_{I^{=}}(\cdot)$ for $g_{i}(\cdot), i \in I^{=}$. Now we prove weak duality theorem which is similar to [9, Theorem 5].

Theorem 4.1 (weak duality). Suppose that $x \in F$ and $(u, \lambda, y) \in F_{D}$ and $f_{i}, i \in M, g_{j}, j \in$ $P$, are generalized convex functions at $u$. Then the following cannot hold:

$$
\begin{gathered}
f_{j}(x) \leq f_{j}(u)+y^{t} g(u), \quad \forall j \in M, \\
f_{i}(x)<f_{i}(u)+y^{t} g(u), \quad \text { for some } i \in M .
\end{gathered}
$$

Proof. Let $x$ be feasible solution for $(M P)$ and let $(\mu, \lambda, y)$ be feasible solution for $(D M)$. 
Suppose contrary to the result that (4.1) hold. Then

$$
\sum_{i \in M} \lambda_{i} f_{i}(x)<\sum_{i \in M} \lambda_{i} f_{i}(u)+y^{t} g(u)
$$

By feasibility of $(u, \lambda, y)$, there exist $\xi_{i} \in \partial_{c} f_{i}(u), i \in M, \eta_{i} \in \partial_{c} g_{i}(u), i \in P$, and $d \in$ $-N_{C}(u)$, such that

$$
\sum_{i \in M} \lambda_{i} \xi_{i}+\sum_{i \in P} y_{i} \eta_{i}=d
$$

Then

$$
\begin{aligned}
\sum_{i \in M} \lambda_{i}\left[f_{i}(x)-\left(f_{i}(u)+y^{t} g(u)\right)\right] & =\sum_{i \in M} \lambda_{i} f_{i}(x)-\sum_{i \in M} \lambda_{i} f_{i}(u)-y^{t} g(u) \\
& \geq(x-u)^{t} \sum_{i \in M} \lambda_{i} \xi_{i}-y^{t} g(u) \\
& =-(x-u)^{t} \sum_{i \in P} y_{i} \eta_{i}-y^{t} g(u)+(x-u)^{t} d \\
& \geq \sum_{i \in P} y_{i}\left(g_{i}(u)-g_{i}(x)\right)-y^{t} g(u) \\
& =-y^{t} g(x) \geq 0,
\end{aligned}
$$

which is a contradiction to (4.2).

Theorem 4.2 (strong duality). If $x^{*}$ is an efficient solution for (MP) and weak duality theorem (Theorem 4.1) holds between (MP) and (DM) and also $g_{i}, i \in P$, are generalized strictly quasiconvex at $x^{*}$, then there exist $y_{i}^{*}, i \in P, \lambda_{i}^{*}>0, i \in M$, such that $\left(x^{*}, \lambda^{*}, y^{*}\right)$ is efficient for (DM) and the objective values of $(M P)$ and $(D M)$ are equal.

Proof. Since $x^{*}$ is an efficient solution for $(M P)$, then by Theorem 3.7 there exist $\lambda_{i}^{*}>$ $0, i \in M$, and $y_{i}^{*} \geq 0, i \in I^{<}\left(x^{*}\right)$, and $d \in-N_{C}\left(x^{*}\right)$ such that (3.15) is satisfied. By taking $y_{i}^{*}=0$ for $i \notin I^{<}\left(x^{*}\right),\left(x^{*}, \lambda^{*}, y^{*}\right)$ is feasible for $(D M)$. Suppose that $\left(x^{*}, \lambda^{*}, y^{*}\right)$ is not efficient for $(D M)$, then there exists $(u, \lambda, y)$ feasible for $(D M)$ such that

$$
\begin{array}{cc}
f_{i}(u)+y^{t} g(u)>f_{i}\left(x^{*}\right)+y^{*} \operatorname{tg}\left(x^{*}\right), & \text { for some } i, \\
f_{j}(u)+y^{t} g(u) \geq f_{j}\left(x^{*}\right)+y^{* t} g\left(x^{*}\right), & \forall j \in M .
\end{array}
$$

However, since $y^{* t} g\left(x^{*}\right)=0$, this would contradict weak duality. The objectives values of $(M P)$ and $(D M)$ are clearly equal at their respective efficient points.

We consider the following dual problem for the problem $(M P)$ and develop duality theorems without constraint qualification for $(M P)$ where the functions of $(M P)$ are not 
assumed to be convex and differentiable. Consider the dual problem

$$
\begin{gathered}
\max \bar{f}(u)=\left(f_{1}(u), \ldots, f_{m}(u)\right) \\
\text { s.t. } \\
0 \in \sum_{i \in M} \lambda_{i} \partial_{c} f_{i}(u)+\sum_{i \in P} y_{i} \partial_{c} g_{i}(u)+N_{C}(u), \\
y_{i} g_{i}(u) \geq 0, \quad i \in P, \\
g_{I^{=}}(u)=0, \\
y_{i} \geq 0, \quad i=1,2, \ldots, p, \\
\lambda_{i}>0, \quad i=1,2, \ldots, m, \\
\sum_{i \in M} \lambda_{i}=1 .
\end{gathered}
$$

Here $F_{D 2}$ denotes the set of feasible solutions to $(D 2 M)$.

Theorem 4.3 (weak duality). Suppose that $x \in F$ and $(u, \lambda, y) \in F_{D 2}$. If $f_{i}, i \in M$, are generalized strictly quasiconvex functions and $g_{i}, i \in P$, are generalized quasiconvex at $u$, then the following cannot hold:

$$
\begin{gathered}
f_{j}(x) \leq f_{j}(u), \quad \forall j \in M, \\
f_{i}(x)<f_{i}(u), \quad \text { for some } i \in M .
\end{gathered}
$$

Proof. Suppose, contrary to the result, that (4.6) hold. Then

$$
\begin{gathered}
f_{j}(x) \leq f_{j}(u), \quad \forall j \in M, \\
f_{i}(x)<f_{i}(u), \quad \text { for some } i \in M .
\end{gathered}
$$

By assumption on $f_{i}, i \in M$, and $g_{i}, i \in P$, we have

$$
\begin{gathered}
\left\langle\sum_{i \in M} \lambda_{i} \xi_{i}, x-u\right\rangle<0, \quad \forall \xi_{i} \in \partial_{c} f_{i}(u), \\
\left\langle\sum_{j \in P} y_{j} \eta_{j}, x-u\right\rangle \leq 0, \quad \forall \eta_{j} \in \partial_{c} g_{j}(u) .
\end{gathered}
$$

This implies that

$$
\left\langle\sum_{i \in M} \lambda_{i} \xi_{i}+\sum_{j \in P} y_{j} \eta_{j}, x-u\right\rangle<0,
$$


for all $\xi_{i} \in \partial_{c} f_{i}(u)$ and $\eta_{j} \in \partial_{c} g_{j}(u)$. From the constraints of $(D 2 M)$, it follows that for some $d \in-N_{C}(u), \xi_{i} \in \partial_{c} f_{i}(u)$, and $\eta_{j} \in \partial_{c} g_{j}(u)$,

$$
\left\langle\sum_{i \in M} \lambda_{i} \xi_{i}+\sum_{j \in P} y_{j} \eta_{j}, x-u\right\rangle \geq(x-u)^{t} d \geq 0 .
$$

This is a contradiction to (4.9).

Theorem 4.4 (strong duality). If $x^{*}$ is an efficient solution for (MP) and weak duality theorem (Theorem 4.3) holds between (MP) and (D2M) and also $g_{i}, i \in P$, are generalized strictly quasiconvex at $x^{*}$, then there exist $y_{i}^{*}, i \in P, \lambda_{i}^{*}>0, i \in M$, such that $\left(x^{*}, \lambda^{*}, y^{*}\right)$ is efficient for (D2M) and the objective values of $(M P)$ and $(D 2 M)$ are equal.

Proof. Since $x^{*}$ is efficient for $(M P)$, then by Theorem 3.7 there exist $\lambda_{i}^{*}>0, i \in M$, and $y_{i}^{*} \geq 0, i \in I^{<}\left(x^{*}\right)$, and $d \in-N_{C}\left(x^{*}\right)$ such that (3.15) is satisfied. By taking $y_{i}^{*}=0$ for $i \notin I^{<}\left(x^{*}\right),\left(x^{*}, \lambda^{*}, y^{*}\right)$ is feasible for $(D 2 M)$. suppose that $\left(x^{*}, \lambda^{*}, y^{*}\right)$ is not efficient for $(D 2 M)$, then there exists $(u, \lambda, y)$ feasible for $(D 2 M)$ such that

$$
\begin{gathered}
f_{i}(u)>f_{i}\left(x^{*}\right), \quad \text { for some } i, \\
f_{j}(u) \geq f_{j}\left(x^{*}\right), \quad \forall j \in M .
\end{gathered}
$$

However, since $x^{*}$ is efficient for $(M P)$, this would contradict weak duality. The objectives of $(M P)$ and $(D 2 M)$ are equal at their respective efficient points.

\section{Acknowledgment}

This work was supported by Project 821134 and by Center of Excellence for Mathematics, University of Isfahan, Isfahan, Iran.

\section{References}

[1] M. S. Bazaraa, H. D. Sherali, and C. M. Shetty, Nonlinear Programming. Theory and Algorithms, John Wiley \& Sons, New York, 1993.

[2] V. Chankong and Y. Y. Haimes, Multiobjective Decision Making. [Theory and Methodology], North-Holland Series in System Science and Engineering, vol. 8, North-Holland, New York, 1983.

[3] F. H. Clarke, Optimization and Nonsmooth Analysis, Canadian Mathematical Society Series of Monographs and Advanced Texts, John Wiley \& Sons, New York, 1983.

[4] F. H. Clarke, Yu. S. Ledyaev, R. J. Stern, and P. R. Wolenski, Nonsmooth Analysis and Control Theory, Graduate Texts in Mathematics, vol. 178, Springer, New York, 1998.

[5] R. R. Egudo, T. Weir, and B. Mond, Duality without constraint qualification for multiobjective programming, Journal of the Australian Mathematical Society. Series B: Applied Mathematics 33 (1992), no. 4, 531-544.

[6] V. Jeyakumar, On optimality conditions in nonsmooth inequality constrained minimization, $\mathrm{Nu}-$ merical Functional Analysis and Optimization 9 (1987), no. 5-6, 535-546.

[7] O. L. Mangasarian and S. Fromovitz, The Fritz John necessary optimality conditions in the presence of equality and inequality constraints, Journal of Mathematical Analysis and Applications 17 (1967), 37-47. 
[8] T. Weir and B. Mond, Duality for generalized convex programming without a constraint qualification, Utilitas Mathematica 31 (1987), 233-242.

[9] ___ Multiple objective programming duality without a constraint qualification, Utilitas Mathematica 39 (1991), 41-55.

[10] P. Wolfe, A duality theorem for non-linear programming, Quarterly of Applied Mathematics 19 (1961), 239-244.

S. Nobakhtian: Department of Mathematics, University of Isfahan, Isfahan 81745-163, Iran

E-mail address: nobakht@math.ui.ac.ir 


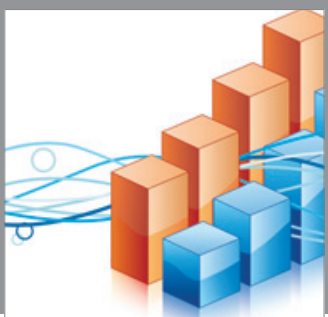

Advances in

Operations Research

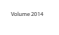

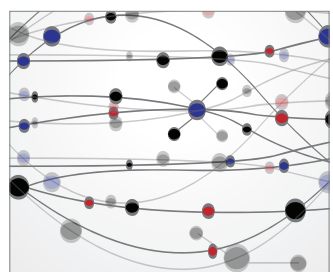

\section{The Scientific} World Journal
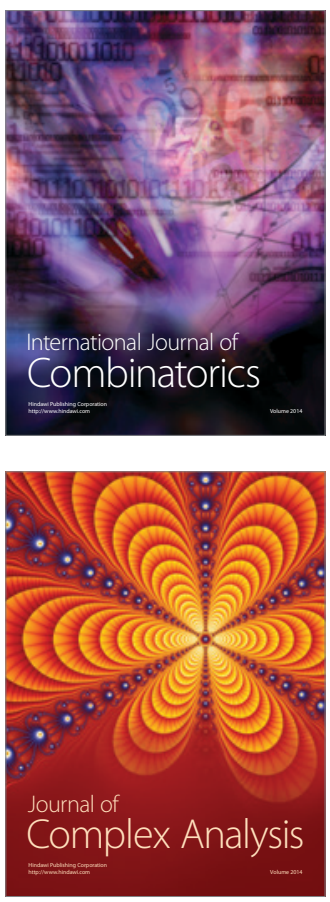

International Journal of

Mathematics and

Mathematical

Sciences
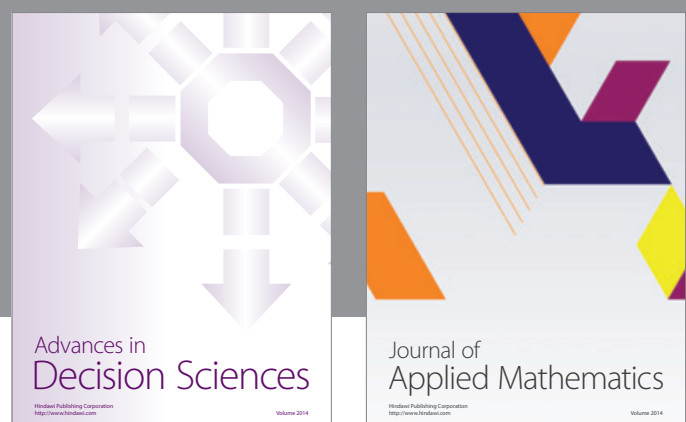

Journal of

Applied Mathematics
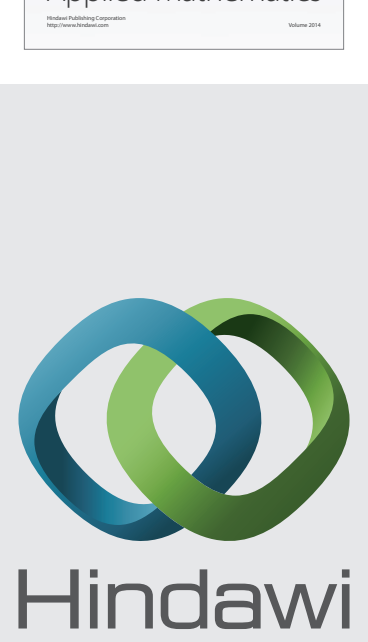

Submit your manuscripts at http://www.hindawi.com
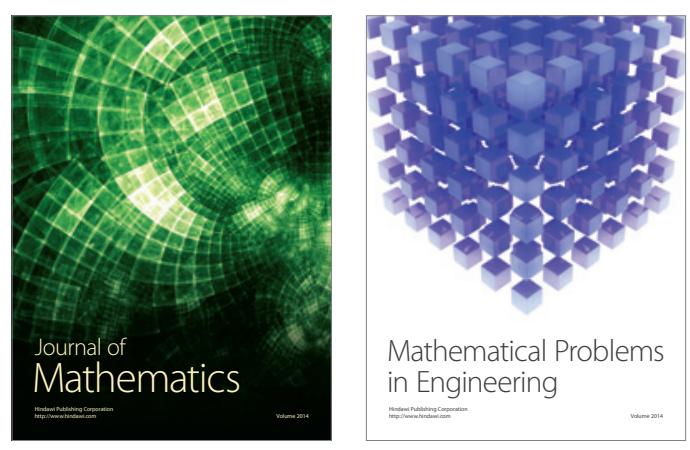

Mathematical Problems in Engineering
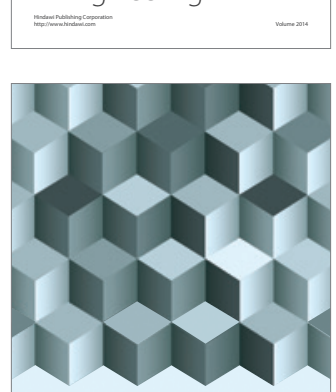

Journal of

Function Spaces
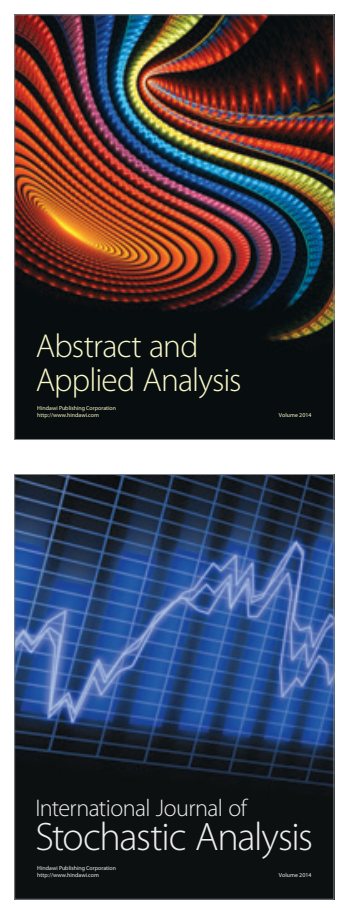

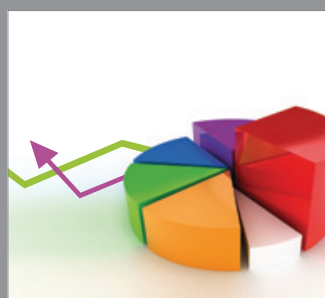

ournal of

Probability and Statistics

Promensencen
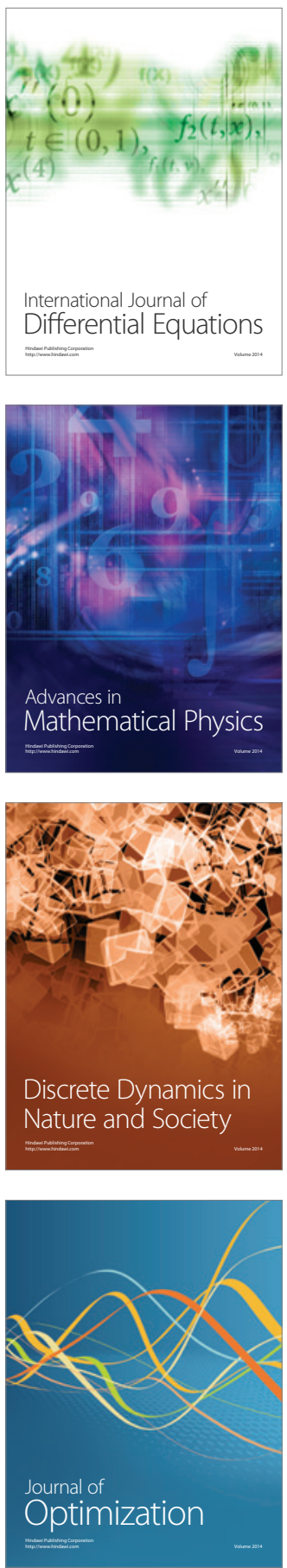